\title{
Truly two-dimensional black holes under dimensional transitions of spacetime
}

\author{
Wanpeng Tan* \\ Department of Physics, Institute for Structure and Nuclear Astrophysics (ISNAP), \\ University of Notre Dame, Notre Dame, Indiana 46556, USA
}

(Dated: March 29, 2021)

A sufficiently massive star in the end of its life will inevitably collapse into a black hole as more deconfined degrees of freedom make the core ever softer. One possible way to avoid the singularity in the end is by dimensional phase transition of spacetime. Indeed, the black hole interior, two-dimensional in nature, can be described well as a perfect fluid of free massless Majorana fermions and gauge bosons under a 2-d supersymmetric mirror model with new understanding of emergent gravity from dimensional evolution of spacetime. In particular, the 2-d conformal invariance of the black hole gives rise to desired consistent results for the interior microphysics and structures including its temperature, density, and entropy.

Essay written for the Gravity Research Foundation 2021 Awards for Essays on Gravitation

The idea of no light escaping from a sufficiently massive astronomical object like a black hole $(\mathrm{BH})$ was first proposed more than two hundred years ago by Michell and Laplace [1, 2]. Shortly after Einstein developed his theory of general relativity (GR), Schwarzschild obtained the first modern BH solution of Einstein's equations in vacuum [3]. The apparent singularity of the solution at the event horizon $r=2 G M$ does not seem to be physical and can be removed in suitable coordinates. But the singularity at $r=0$ is genuine indicating that GR breaks down under such extreme conditions and new physics may be needed to describe the $\mathrm{BH}$ interior within its event horizon.

In the following, we will apply recently developed supersymmetric mirror models (SMM) and new understanding of gravity as an emergent classical phenomenon due to dimensional phase transitions of spacetime to study such extreme celestial objects. In particular, the interior of a black hole, two-dimensional in nature, can be elegantly described by the 2-d model SMM2.

The existence of a mirror sector of the Universe has been conjectured since Lee and Yang published their Nobel Prize-winning work on parity violation [4]. It is conceivable that there exist two sectors of particles sharing the same gravity but governed by two separate gauge groups under 4-d spacetime [5]. Some early works on mirror matter theory had discussed interesting perspectives mainly in cosmology [6] 8]. Later attempts to introduce ad hoc feeble interactions between the two sectors might be too conservative [9-11. Most recent works [12 22, with new understanding of mirror symmetry, supersymmetry (SUSY), and dimensional phase transitions of spacetime can consistently and quantitatively solve a variety of puzzles in fundamental physics and cosmology and may indeed lead us to new physics beyond the Standard Model we have been looking for.

* wtan@nd.edu 


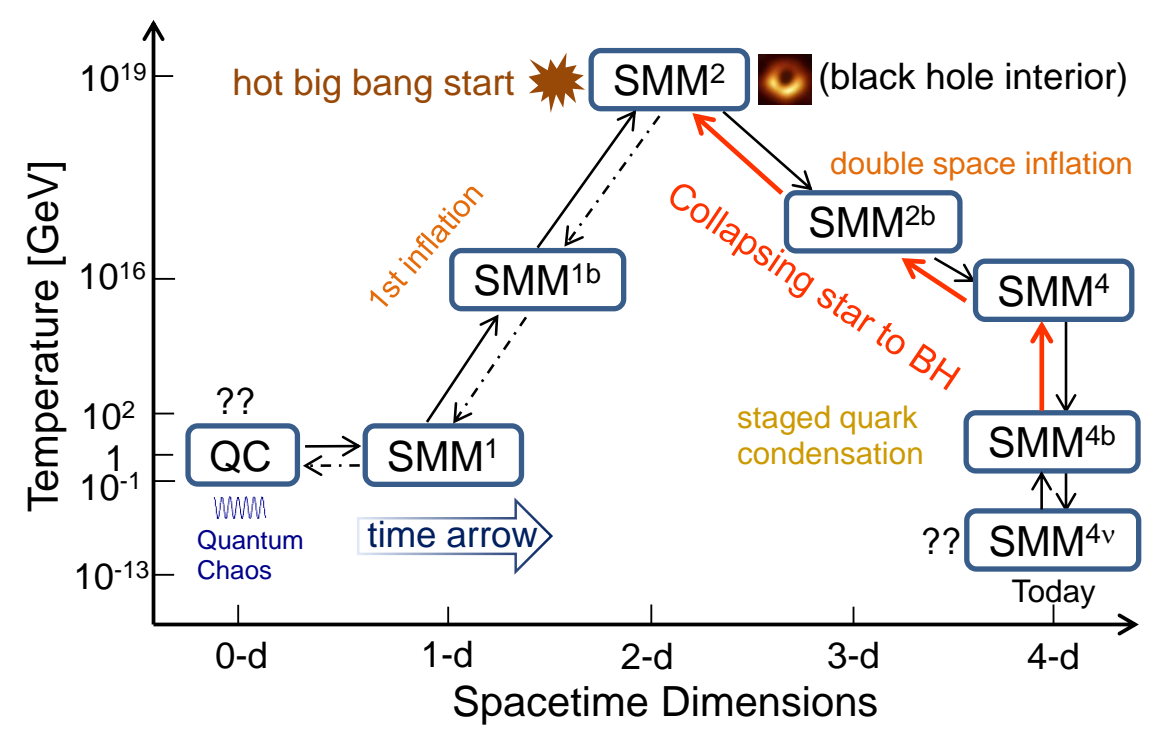

FIG. 1. The schematic diagram (not to scale) is shown for the Supersymmetric Mirror Models at various phases of spacetime. Red arrows indicate the path of a collapsing star into a black hole. Adapted from Ref. [22].

The idea [18 22] is that spacetime was first born with one time dimension. Mirror symmetry is nothing but the orientation symmetry of the underlying geometry (same as time reversal symmetry in 1-d). The sole scalar then acquired mass in the spontaneous symmetry breaking mechanism leading to the emergence of the time arrow and another dimension of space in inflation. In the resulting 2-d spacetime, Majorana fermions and $U(1)$ gauge bosons emerged and the hot big bang started at the scale of Planck energy.

As the Universe cooled down, Majorana fermions started to condense leading to the process of double space inflation. Eventually 4 -d spacetime formed at temperature of $\sim 10^{16}$ $\mathrm{GeV}$ and the two scalar inflatons decayed into massless quarks and leptons in two separate sectors, respectively, to reheat the Universe. At the scale of $\sim 10^{2} \mathrm{GeV}$, staged quark condensation started a series of phase transitions including electroweak and QCD phase transitions. As the Universe became cold enough, the ordinary sector has formed stars and galaxies while the mirror sector serves as the dark matter observed today. Such a picture under the proposed Supersymmetric Mirror Models is depicted in Fig. (1).

The reverse process from SMM4b to SMM2 as shown in Fig. (1) could also occur for a massive star to collapse into a black hole from 4-d to 2-d. The underlying model (SMM2) in 2-d spacetime has the on-shell Lagrangian [19, 20],

$$
\mathcal{L}_{\mathrm{SMM} 2}=-\frac{1}{4} F_{\mu \nu} F^{\mu \nu}+\frac{i}{2}\left(\lambda_{L}^{\dagger} \bar{\sigma}^{\mu} \partial_{\mu} \lambda_{L}+\lambda_{R}^{\dagger} \sigma^{\mu} \partial_{\mu} \lambda_{R}\right)
$$

where $F_{\mu \nu}=\partial_{\mu} A_{\nu}-\partial_{\nu} A_{\mu}$ is the $U(1)$ gauge tensor and the Majorana fermion $\lambda$ has to be neutral and does not couple to the gauge field $A_{\mu}$. Both $\lambda$ and $A_{\mu}$ are massless and have two components or degrees of freedom $\left(n_{f}=n_{b}=2\right)$. They form the simplest $N=1$ abelian gauge SUSY multiplet $(1,1 / 2)$. As we shall use later, the effective number of relativistic degrees of freedom $g^{*}=n_{b}+1 / 2 n_{f}=3$.

On the other hand, gravity in 2-d is described by the smooth spacetime geometry as follows [21],

$$
\mathcal{R}+2 \Lambda=-8 \pi G T_{\mu}^{\mu}=0
$$


where $\mathcal{R}$ is the Ricci curvature scalar, $\Lambda$ is the cosmological constant, and the energymomentum tensor $T_{\mu}^{\mu}$ has to be traceless. In SMM2 as shown in Eq. (1), free massless Majorana fermions and $U(1)$ gauge bosons make a perfect fluid resulting in the vanishing trace of $T_{\mu \nu}$ and therefore is consistent with the 2-d gravity / spacetime model of Eq. (2).

Eqs. (12) together describe a conformally invariant 2-d quantum and spacetime theory. Its effective number of relativistic degrees of freedom $g^{*}=3$ is identically the central charge $c$ of the Virasoro symmetry algebra in 2-d conformal field theory (CFT) 223. The corresponding Weyl anomaly in 2-d CFT 24] can produce a non-vanishing trace of $2 \rho_{\Lambda}=-c /(24 \pi G) \mathcal{R}$ due to nontrivial metric, where $\rho_{\Lambda}$ can be interpreted as vacuum energy density and related to the cosmological constant via $\Lambda=8 \pi G \rho_{\Lambda}$. This trace anomaly, introduced dynamically by the inflaton scalars of Majorana condensates [19], is related to a topological invariant - the Euler characteristic [25], which may be important for studying nontrivial spacetime topology during the double space inflation process SMM2b [19].

However, for a static spherically symmetric black hole, its interior should be Ricci-flat, i.e, $\mathcal{R}=0$ and can then be studied in 2-d CFT on a torus. The Euler characteristic of a torus vanishes and makes it consistent that the black hole interior should be static and free of the trace anomaly or $\Lambda=0$. The general solution of the 2-d metric can then be written as,

$$
d s^{2}=e^{2 \omega(x)}\left(d t^{2}-d x^{2}\right)
$$

where $\omega(x)$ defines the conformal transformations of the metric.

We will present the views of the $\mathrm{BH}$ interior by both a distant exterior observer and an interior one. The two reference frames are connected by the global scale transformation under 2-d CFT. There are two quantities that the two observers see the same: proper energy density $\rho$ and total entropy $S_{\mathrm{BH}}$.

The view of the exterior observer is fairly simple for a Schwarzschild black hole with mass $M$ and radius $R=2 G M$. The $\mathrm{BH}$ interior can be regarded as a 2 -d torus with two circumferences of $2 R$ (space) and $2 \pi R$ (time), which can also be viewed externally as a dual of the event horizon surface, notably with the same area $A=4 \pi R^{2}$. Its conformally invariant 2-d energy density is,

$$
\rho=\frac{M}{2 R}=\frac{1}{4 G}
$$

which is the same as the CFT Casimir energy density $\rho_{c}=E_{c} /(2 R)=c /(12 G)$ with no negative sign under the condition of $c=g^{*}=3$. This 2-d structure with a constant energy density provides a natural explanation of the linear relation between mass and radius of the black hole.

The entropy of an $n+1$ dimensional CFT on $R \times S^{n}$ can be given by the Cardy-Verlinde formula [26, 27],

$$
S=\frac{2 \pi R}{n} \sqrt{E_{c}\left(2 E-E_{c}\right)}
$$

where $E_{c}=2 R c /(12 G)$ is the Casimir energy in 2-d CFT (i.e., $\left.n=1\right)$ and the total energy $E=2 R \rho$ is exactly the same as $E_{c}$ if the central charge $c=g^{*}=3$. Under the $\mathrm{BH}$ conditions of $n=1$ and $c=g^{*}=3$, Eq. (5) becomes the well-known Bekenstein-Hawking entropy [28, 29],

$$
S_{\mathrm{BH}}=\frac{A}{4 G} .
$$


Based on Maldacena's AdS/CFT correspondence [30] and Witten's arguments [31, Verlinde proposed a more general entropy formula for the same CFT on $R \times S^{n}$ as [27],

$$
S=\frac{c A}{12 n G}
$$

which again gives the same entropy as Eq. (6) for $n=1$ and $c=g^{*}=3$.

The BH entropy can also have an equivalent thermodynamic explanation. Namely, the exterior observer sees no free energy from the black hole, i.e., its partition function $Z=1$. As such, the entropy is solely determined by its total energy / mass $M$ and temperature $1 / \beta$ where $\beta=2 \pi R$ is the Euclidean time period or the time circumference of the torus. Then, the same BH entropy can be easily calculated from

$$
S_{\mathrm{BH}}=\ln (Z)+\beta M=\beta M=\frac{A}{4 G}
$$

where, notably, the interior temperature of $1 / \beta$ viewed by the exterior observer is twice as high as the surface temperature that is responsible for Hawking radiation [29, 32].

From the view of the interior observer, the $\mathrm{BH}$ interior is composed of a perfect fluid of Majorana fermions and gauge bosons governed by SMM2. Its energy and entropy densities in 2-d spacetime can be obtained as,

$$
\begin{aligned}
& \rho=\frac{\pi}{6} g^{*} T_{\mathrm{in}}^{2}=\frac{\pi}{2} T_{\mathrm{in}}^{2} \\
& s=\frac{\rho}{T_{\mathrm{in}}}=\frac{\pi}{2} T_{\mathrm{in}}
\end{aligned}
$$

where $T_{\text {in }}$ is the interior temperature. Such an expression of 2-d entropy density in Eq. (10) has also been obtained in general 2-d CFT in the high temperature limit [26, 33.

Using the two views of the energy density in Eqs. (4) 9 ), we can obtain the temperature of the $\mathrm{BH}$ interior,

$$
T_{\mathrm{in}}=\frac{1}{\sqrt{2 \pi G}} \simeq \frac{0.4}{\sqrt{G}}
$$

which is constant and just below the Planck energy of $1 / \sqrt{G} \sim 10^{19} \mathrm{GeV}$ but well above the double space inflation energy scale of $10^{16} \mathrm{GeV}$. This means that the interior temperature does meet the criteria for SMM2 in 2-d spacetime and this BH model is self-consistent. The constant energy density and temperature also indicate that such $\mathrm{BH}$ interior structures are indeed stable.

As viewed by the interior observer, the torus is conformally dilated as follows,

$$
2 R \rightarrow L_{s} \equiv e^{\omega} 2 R, \quad 2 \pi R \rightarrow L_{t} \equiv e^{-\omega} 2 \pi R
$$

where $L_{s}$ and $L_{t}$ are the different space and time scales (or sizes of the torus) for the interior observer under the global dilation or scale transformation. From $L_{t}=1 / T_{\text {in }}$, we can obtain the dilation factor as,

$$
e^{\omega}=\sqrt{\frac{2 \pi}{G}} R \simeq 4.58 \times 10^{38} \frac{M}{M_{\odot}} .
$$

It is now easy to calculate the entropy from the point of view of the interior observer,

$$
S_{\mathrm{BH}}=e^{\omega} 2 R s=\frac{A}{4 G}
$$


which is the same Bekenstein-Hawking entropy. To demonstrate the enormous BH size seen by the interior observer, we can calculate it for one solar mass to be $e^{\omega} 2 R \sim 10^{42} \mathrm{~m}$ in contrast to the size of the observable Universe $\left(10^{27} \mathrm{~m}\right)$.

In summary, black holes are presented to be 2-d in nature under the picture of dimensional transitions of spacetime. The apparent singularity at the event horizon under 4-d general relativity is most likely topological describing a transition from the exterior 4-d spacetime to the interior 2-d spacetime. Techniques especially topological ones developed in studies of renormalization group, loop quantum gravity, and other quantum gravity approaches could be applied around the event horizon and possibly elsewhere such as the space inflation process of the early Universe.

\section{ACKNOWLEDGMENTS}

This work is supported in part by the National Science Foundation under grant No. PHY-2011890. Funding from the faculty research support program at the University of Notre Dame is also acknowledged.

[1] P.-S. Laplace, Exposition Du Systeme Du Monde, 1st ed. (Cercle-Social, 1796).

[2] J. Michell, On the means of discovering the distance, magnitude, \&c. of the fixed stars, in consequence of the diminution of the velocity of their light, in case such a diminution should be found to take place in any of them, and such other data should be procured from observations, as would be farther necessary for that purpose., Philos. Trans. R. Soc. Lond. 74, 35 (1784).

[3] K. Schwarzschild, über das Gravitationsfeld eines Massenpunktes nach der Einsteinschen Theorie, Sitzungsberichte K. Preußischen Akad. Wiss. Berl. , 189 (1916); On the gravitational field of a mass point according to Einstein's theory, arXiv:physics/9905030 (1999), arXiv:physics/9905030.

[4] T. D. Lee and C. N. Yang, Question of Parity Conservation in Weak Interactions, Phys. Rev. 104, 254 (1956).

[5] I. Y. Kobzarev, L. B. Okun, and I. Y. Pomeranchuk, On the possibility of experimental observation of mirror particles, Sov J Nucl Phys 3, 837 (1966).

[6] S. I. Blinnikov and M. Y. Khlopov, Possible Astronomical Effects of Mirror Particles, Sov. Astron. 27, 371 (1983).

[7] E. W. Kolb, D. Seckel, and M. S. Turner, The shadow world of superstring theories, Nature 314, 415 (1985).

[8] H. M. Hodges, Mirror baryons as the dark matter, Phys. Rev. D 47, 456 (1993).

[9] Z. Berezhiani and L. Bento, Neutron-Mirror-Neutron Oscillations: How Fast Might They Be?, Phys. Rev. Lett. 96, 081801 (2006).

[10] J.-W. Cui, H.-J. He, L.-C. Lü, and F.-R. Yin, Spontaneous mirror parity violation, common origin of matter and dark matter, and the LHC signatures, Phys. Rev. D 85, 096003 (2012).

[11] R. Foot, Mirror dark matter: Cosmology, galaxy structure and direct detection, Int. J. Mod. Phys. A 29, 1430013 (2014).

[12] W. Tan, Neutron oscillations for solving neutron lifetime and dark matter puzzles, Phys. Lett. B 797, 134921 (2019), arXiv:1902.01837. 
[13] W. Tan, Neutron-mirror neutron oscillations in stars (2019), arXiv:1902.03685 [astro-ph, physics:hep-th, physics:nucl-ex].

[14] W. Tan, Neutron-mirror neutron oscillations for solving the puzzles of ultrahigh-energy cosmic rays (2019), arXiv:1903.07474 [astro-ph, physics:hep-ph].

[15] W. Tan, Kaon oscillations and baryon asymmetry of the universe, Phys. Rev. D 100, 063537 (2019), arXiv:1904.03835.

[16] W. Tan, Laboratory tests of the ordinary-mirror particle oscillations and the extended CKM matrix (2019), arXiv:1906.10262 [hep-ex, physics:hep-ph, physics:nucl-ex].

[17] W. Tan, Invisible decays of neutral hadrons (2020), arXiv:2006.10746 [hep-ph].

[18] W. Tan, Dark energy and spontaneous mirror symmetry breaking (2019), arXiv:1908.11838 [gr-qc, physics:hep-ph, physics:hep-th].

[19] W. Tan, Hierarchy of Supersymmetric Mirror Models and Dimensional Evolution of Spacetime Preprint: https://osf.io/8qawc (Open Science Framework, 2020).

[20] W. Tan, No single unification theory of everything (2020), arXiv:2003.04687 [physics],

[21] W. Tan, From Neutron and Quark Stars to Black Holes, Preprint: https://osf.io/2jywx (Open Science Framework, 2020).

[22] W. Tan, First Principles of Consistent Physics, Preprint: https://osf.io/cj94u (2021).

[23] P. Di Francesco, P. Mathieu, and D. Sénéchal, Conformal Field Theory, Graduate Texts in Contemporary Physics (Springer, New York, 1997).

[24] S. Deser, M. J. Duff, and C. J. Isham, Non-local conformal anomalies, Nucl. Phys. B 111, 45 (1976); M. J. Duff, Twenty years of the Weyl anomaly, Class. Quantum Grav. 11, 1387 (1994).

[25] M. J. Duff, Observations on conformal anomalies, Nuclear Physics B 125, 334 (1977).

[26] J. L. Cardy, Operator content of two-dimensional conformally invariant theories, Nucl. Phys. B 270, 186 (1986).

[27] E. Verlinde, On the Holographic Principle in a Radiation Dominated Universe (2000), arXiv:hep-th/0008140.

[28] J. D. Bekenstein, Black Holes and Entropy, Phys. Rev. D 7, 2333 (1973).

[29] S. W. Hawking, Particle creation by black holes, Commun. Math. Phys. 43, 199 (1975).

[30] J. M. Maldacena, The Large N Limit of Superconformal Field Theories and Supergravity, Adv. Theor. Math. Phys. 2, 231 (1998), arXiv:hep-th/9711200.

[31] E. Witten, Anti-de Sitter Space, Thermal Phase Transition, And Confinement In Gauge Theories, Adv. Theor. Math. Phys. 2, 505 (1998), arXiv:hep-th/9803131.

[32] S. W. Hawking, Black hole explosions?, Nature 248, 30 (1974).

[33] I. Affleck, Universal term in the free energy at a critical point and the conformal anomaly, Phys. Rev. Lett. 56, 746 (1986). 